\title{
Article \\ Study of Possible Frequency Dependence of Small AC Fields on Magnetic Flux Trapping in Niobium by Polarized Neutron Imaging
}

\author{
Wolfgang Treimer ${ }^{1, *}$, Tobias Junginger $2,3\left(1\right.$ and Oliver Kugeler ${ }^{4}$ \\ 1 Department Mathematics, Physics \& Chemistry, University of Applied Sciences Berlin, Berliner Hochschule \\ für Technik Berlin, D-13353 Berlin, Germany \\ 2 Department of Physics and Astronomy, University of Victoria, Victoria, BC V8P 5C2, Canada; \\ junginger@uvic.ca \\ 3 TRIUMF, 4004 Wesbrook Mall, Vancouver, BC V6T 2A3, Canada \\ 4 Helmholtz-Zentrum Berlin für Materialien und Energie GmbH (HZB), Hahn-Meitner Platz 1, \\ D-14109 Berlin, Germany; oliver.kugeler@helmholtz-berlin.de \\ * Correspondence: treimer@beuth-hochschule.de
}

check for updates

Citation: Treimer, W.; Junginger, T.; Kugeler, O. Study of Possible Frequency Dependence of Small AC Fields on Magnetic Flux Trapping in Niobium by Polarized Neutron Imaging. Appl. Sci. 2021, 11, 6308. https://doi.org/10.3390/app11146308

Academic Editor: Serghei Klimin

Received: 15 May 2021

Accepted: 5 July 2021

Published: 8 July 2021

Publisher's Note: MDPI stays neutral with regard to jurisdictional claims in published maps and institutional affiliations.

Copyright: (C) 2021 by the authors Licensee MDPI, Basel, Switzerland. This article is an open access article distributed under the terms and conditions of the Creative Commons Attribution (CC BY) license (https:// creativecommons.org/licenses/by/ $4.0 /)$.
Abstract: Reducing the size of ambient magnetic flux trapping during cooldown in superconducting radio-frequency niobium cavities is essential to reaching the lowest power dissipation as required for continuous wave application. Here, it is suggested that applying an alternating magnetic field superimposed to the external DC field can potentially reduce the size of trapped flux by supporting flux line movement. This hypothesis is tested for the first time systematically on a buffered chemically polished (BCP) niobium sample before and after high temperature annealing, a procedure which is known to reduce flux pinning. External low-frequency (Hz-range) magnetic fields were applied to the samples during their superconducting transition and the effect of varying their amplitude, frequency and offset was investigated. A few results can be highlighted: The influence of the frequency and magnitude of the $\mathrm{AC}$ fields on the flux trapping in the untreated $\mathrm{Nb}$ sample cannot be neglected. The trapped flux seems to be homogeneously distributed, unlike the flux trapping in, e.g., lead $(\mathrm{Pb})$, which is a type I superconductor. After annealing, the $\mathrm{Nb}$ sample shows practically no dependency of flux trapping on external AC fields. The trapped magnetic flux was measured by polarized neutron imaging, and calculations of trapped fields show good agreement with experimental results.

Keywords: SRF; superconductivity; niobium; meissner effect; trapped flux; polarized neutron imaging

\section{Introduction}

Superconducting radio-frequency (SRF) cavities consisting of Niobium are enabling components of many modern particle accelerators from spallation neutron sources to $\mathrm{CW}$ (continuous wave) free electrons $\mathrm{X}$-ray lasers. As $\mathrm{CW}$ applications become more and more important and repetition rates are increasing to obtain higher beam currents, the power dissipation in the cavity walls becomes a major limiting factor not only from a maximumperformance point of view, but also with respect to cost minimization. In the attempt to push superconducting materials to elevated performance, the degrading impact of trapped magnetic vortices must be investigated, understood and, ideally, eliminated. Over the past few years, several studies demonstrated in samples, in cavities and also in module-like operation how beneficial a reduction in trapped magnetic flux is. In particular, it was found that the size of trapped flux is affected by several different parameters, such as the cooldown conditions or material properties like RRR (residual resistivity ratio). This laid the basis for further, systematic investigations [1-11]. These methods have in common that the magnetic flux is observed indirectly-either by measuring its impact on the cavity performance or by measuring the impact of the incomplete Meissner 
transition on the ambient magnetic field or by small angle neutron scattering. From these indirect measurements one then needs to deduce, what the actual flux distribution in the superconductor is $[2,12-16]$.

In this paper, we first describe a direct method to measure magnetic fields inside the cavity material and then present results of our investigations of the influence of AC external magnetic fields on flux trapping behavior. The Meissner phase is the energetically favorable state of the whole sample, because it is a state of complete expulsion of any ambient magnetic field from the bulk material. Therefore, any deviation from this equilibrium state must have been caused by trapping a number of quantized flux lines within the $\mathrm{Nb}$ material before they could have been driven out.

The idea of superimposing external AC magnetic fields is that a movement of fluxlines over macroscopic distances could be supported by those fields. Flux-lines can move freely in a normal-conducting medium, while they are driven out of superconducting areas. In the mixed state of coexisting superconducting and normal conducting regions, the movement of the flux-lines becomes viscous. The viscosity of the flux-line movement is rapidly changing in the vicinity of the transition temperature. In order to support the expulsion, it is necessary to have the sample at a temperature near or slightly below the transition temperature, where the flux lines are still sufficiently mobile. Furthermore, the driving force for the flux-line movement would have to exceed the blocking force on a flux-line. The movement of the flux-line itself can be suppressed by two mechanisms: (1) flux-trapping, i.e., encirclement of the flux-line by superconducting regions, or (2) fluxpinning, i.e., circumvention of the flux-line movement at features that deviate from the pure $\mathrm{Nb}$ matrix, such as dislocations or non- $\mathrm{Nb}$ inclusions. The mobility of the flux lines is highest at $\mathrm{T} \sim \mathrm{Tc}$, an alternating magnetic field applied to the sample during cooling is supposed to enhance the mobility of the flux lines and their movement in the axial direction by adding a constant field.

In the frame of a series of previous experiments, different sample treatments such as heat treatment, spatial temperature gradients and different cooling rates have been tested with respect to their capability to minimize flux trapping. It could be shown that a recrystallizing heat treatment considerably reduces trapped flux. At the same time, it was observed that single crystal $\mathrm{Nb}$ samples trap less flux than polycrystalline samples $[2,17,18]$. In these experiments, trapped magnetic fields after field cooling (FC) could be identified as different for untreated and treated samples, but also seemed to depend on an applied external AC field. This led to the systematic study of the influence of flux trapping on external fields presented here. One of the basic problems is a reliable determination of flux trapping. We use a complementary method, polarized neutron imaging, which enables us to determine the trapped field directly and which provides spatially resolved in-depth information from the entire volume of the investigated sample [17,19-25]. In order to understand our investigations, some basics of polarized neutron imaging are given.

\section{Short Theory of Polarized Neutron Imaging}

It has been shown that polarized neutrons are particularly suitable for visualizing magnetic fields [19-24,26]. Using tomographic methods, shape, size, amount of magnetic fields in superconducting matter in large $\left(\sim \mathrm{cm}^{3}\right)$ samples could be determined [22,27].

Based on absorption, radiography and tomography with (thermal) neutrons are quite similar to well-known X-ray techniques, but due to their magnetic moment $\mu_{n}$ $\left(\mu_{n}=-0.96623651 \times 10^{-26}\left[J T^{-1}\right]\right)$ imaging with polarized neutrons involves in addition the interaction of the neutron spin with a magnetic field. If a neutron enters a magnetic field, its spin starts rotations around B with the so-called Larmor frequency $\omega_{L}=\gamma_{n} B$, $\gamma_{n}=1.83247172 \times 10^{8}\left[T^{-1} S^{-1}\right][28,29]$ (all values see [30-32]). The rotation angle $\phi$ 
depends on the velocity vs. of the neutron and can be used to determine the amount of $B$, calculating the path integral along $B$,

$$
\phi=\omega_{L} t=\gamma_{n} B \cdot t=\frac{\gamma_{n}}{\mathrm{~V}} \int_{p a t h} \vec{B} \cdot d \vec{s}
$$

If 's' is the path length in the sample $\phi$ becomes

$$
\phi=\frac{\gamma_{n} \cdot \lambda \cdot m}{h} \cdot B \cdot s
$$

Assuming the sample placed in a $\{x y z\}$ coordinate system, and considering only two projections $\left(0\right.$ and $\left.90^{\circ}\right)$, the path integral (Equation (1)) then becomes [22]

$$
\begin{aligned}
& R_{0, \alpha}\{B(x, y, z)\}= \\
& \int_{-\infty}^{\infty} \int_{-\infty}^{\infty} B(x, z) \cdot \delta(p-x \cdot \cos (\alpha)-z \cdot \sin (\alpha)) \cdot d x \cdot d z \\
& R_{90, \alpha}\{B(x, y, z)\}= \\
& \int_{-\infty}^{\infty} \int_{-\infty}^{\infty} B(y, z) \cdot \delta(p-y \cdot \cos (\alpha)-z \cdot \sin (\alpha)) \cdot d y \cdot d z
\end{aligned}
$$

$R_{0, \alpha}\{B(x, y, z)\}$ is the $2 \mathrm{D}$-Radon transform of the sample when its rod axis is perpendicular to the $\{x z\}$ plane and $R_{90, \alpha}\{B(x, y, z)\}$ the 2D-Radon transform of the sample when its rod axis is perpendicular to the $\{y z\}$ plane. For $R_{0, \alpha}\{B(x, y, z)\} \alpha$ is constant in this calculation, it is usually the (rotation) angle of the sample with respect either to the $\mathrm{x}$ or $\mathrm{z}$ axis, and for $R_{90, \alpha}\{B(x, y, z)\}$ parallel either to the $\mathrm{y}$ or $\mathrm{z}$ axis. $\mathrm{p}$ is the scanning parameter and describes the parallel beam scanning across a slice. The intensity registered by a $2 \mathrm{D}$ neutron detector is then given as

$$
\begin{aligned}
& I(x, z)=\underbrace{I_{0} \cdot T \cdot \exp \left(-\int_{\text {path }} \Sigma(s) d s\right)}_{I_{\text {att }}(x, z)} \cdot \underbrace{\frac{1}{2}(1+\cos \phi(x, z))}_{I_{\text {spin }}(x, z)} \\
& I(y, z)=\underbrace{I_{0} \cdot T \cdot \exp \left(-\int_{\text {path }} \Sigma(s) d s\right)}_{I_{\text {att }}(y, z)} \cdot \underbrace{\frac{1}{2}(1+\cos \phi(y, z))}_{I_{\text {spin }}(y, z)}
\end{aligned}
$$

$I_{0}$ is the incident intensity, $\Sigma(s)$ the linear attenuation coefficient of the sample, $I_{\text {spin }}$ is the 'magnetic' part of the transmitted intensity $I(x, z)$ and $I(y, z)$, respectively. $I(x, z)$ and $I(y, z)$ are spatially resolved two-dimensional depolarization images. Each trapped magnetic field causes a characteristic depolarization image, which can be calculated from first principles using Equations (2)-(4) and compared with experimental neutron images.

\section{Experiments}

\subsection{Instrument, Samples and Field Cooling (FC)}

The motivation for applying an AC field during FC was given in the introduction, i.e., to support the migration of flux-lines over macroscopic distances by superimposing external alternating magnetic fields. The question to be answered is the influence of external AC fields on flux trapping during FC from $\mathrm{T} \geqslant T_{\mathcal{C}} \rightarrow T<T_{\mathcal{C}}$ and how to measure and to quantize them. Several parameters (purity, crystalline, poly-crystalline, chemically treatment) are relevant in order to associate possible flux trapping to externally applied magnetic AC fields. A high purity $(99.999 \%)$ polycrystalline crystal $\mathrm{Nb}$ sample of cylindrical shape (diameter $=10 \mathrm{~mm}$, height $=5 \mathrm{~mm}$ ) always underwent the same cooling procedure, i.e., cooling was started at equilibrium $\mathrm{T}=15 \mathrm{~K}$ and was ended at $\mathrm{T}=5 \mathrm{~K}$, well below the critical temperature of $\mathrm{Nb}\left(\mathrm{T}_{\mathcal{C}}=9.2 \mathrm{~K}\right)$. The sample first was left 
'untreated' $\left(\mathrm{Nb}=\mathrm{Nb}_{\text {oxid }}\right)$ and after the first series of measurements further treated by buffered chemically polishing $\left(\mathrm{Nb}_{b a k}\right.$, i.e., annealing at $1400{ }^{\circ} \mathrm{C}$ followed by buffered chemically polishing (BCP), removing $150 \mu \mathrm{m}$ of material). Muon spin rotation studies have shown that this treatment is very effective in reducing flux-pinning [33].

During FC different AC fields (B amplitude was $\pm 5 \mathrm{mT}, \mathrm{f}=0.1 \mathrm{~Hz}, 0.25 \mathrm{~Hz}$ and $0.5 \mathrm{~Hz}$ ) and $\mathrm{B}$ offsets were $0 \mathrm{mT} \ldots 5 \mathrm{mT}$ ) were applied to the sample. Reaching $\mathrm{T}=5 \mathrm{~K}$, $\mathrm{B}_{\text {ext }}=\mathrm{B}+\mathrm{B}_{\text {offset }}$ was switched off and neutron images were recorded in $0^{\circ}$ and $90^{\circ}$ sample orientations. Then, the sample was warmed up to $15 \mathrm{~K}$ before starting the next series. This procedure was repeated for each parameter set $\left\{f, B_{\text {ext }}, B_{\text {offset }}\right\}$.

The experiments have been carried out at the instrument PONTO II at the BER II of the Helmholtz Center Berlin, Wannsee (Figure 1). The neutron beam (mean wave length $\lambda=0.32 \mathrm{~nm} \sim 8 \mathrm{meV}$ ) was collimated horizontally and vertically $0.1^{\circ} \sim 1.745 \times 10^{-3} \mathrm{rad}$, corresponding to a $\mathrm{L} / \mathrm{D}=573$ (i.e., divergence $\sim 1.745 \times 10^{-3} \mathrm{rad} \sim 0.1^{\circ}$ ). The neutron beam was polarized with a super mirror, yielding a $92 \%$ beam polarization, the magnetic guide field was parallel to the field direction of the polarizer and the analyzer. In front of the $2 \mathrm{D}$ detector $(2 \mathrm{k} \times 2 \mathrm{k}$, pixel size $=13.5 \mu \mathrm{m})$ a spin analyzer acted similar to an optical polarizer with the difference that the opaque orientation for spin down was $180^{\circ}$. Thus, the transparency of the spin analyzer can change from $100 \%$ for spin up neutrons (spin parallel to spin analyzer) to $0 \%$ for spin down neutrons (spin anti-parallel to spin analyzer), corresponding to a maximum image contrast of $100 \%$ and to a minimum contrast of $0 \%$.

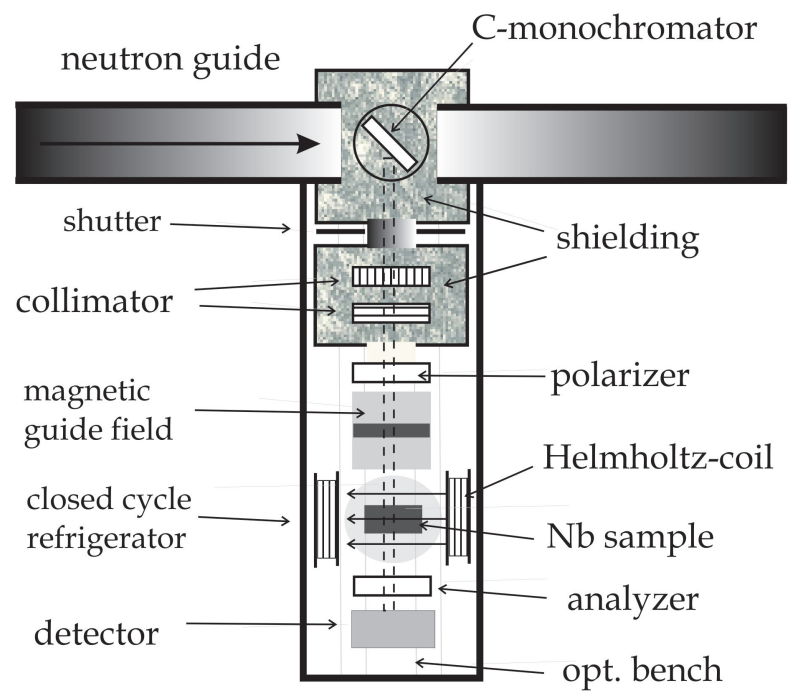

Figure 1. PONTO II Instrument for polarized neutron imaging at the BER II of the University of Applied Sciences Beuth Hochschule fuer Technik Berlin [34].

In the case of unpolarized neutron imaging, the spatial resolution $\left[\Delta_{h} \times \Delta_{v}\right] 60 \mathrm{~mm}$ apart from the converter screen was $55 \mu \mathrm{m} \times 55 \mu \mathrm{m}$; however, in polarized neutrons imaging about $160 \mu \mathrm{m} \times 160 \mu \mathrm{m}$ for a sample-screen distance of $200 \mathrm{~mm}$ [35]. The samples were kept in special Al holder in a conventional cryostat, which was placed between two Helmholtz coils that could generate different external AC magnet fields up to $60 \mathrm{mT}$ and up to $10 \mathrm{~Hz}$.

The sample was cooled down from $15 \mathrm{~K}$ to $5 \mathrm{~K}$ well below $\mathrm{T}_{\mathcal{C}}=9.2 \mathrm{~K}$ as described above, with an external applied field $\mathrm{B}_{\mathrm{ext}}$ always parallel to the rod axis $\left(0^{\circ}\right.$ orientation, (a) in Figure 2). Concerning the cooling rate, a separate series of measurements showed no dependency of flux trapping from cooling speed which was varied using $0.1 \mathrm{~K} / \mathrm{min}$, $0.3 \mathrm{~K} / \mathrm{min}$ and $1 \mathrm{~K} / \mathrm{min}$; therefore, the fastest cooling time $1 \mathrm{~K} / \mathrm{min}$ was used. 
a)

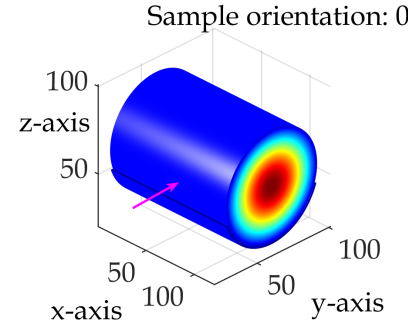

Sample orientation: $90^{\circ}$

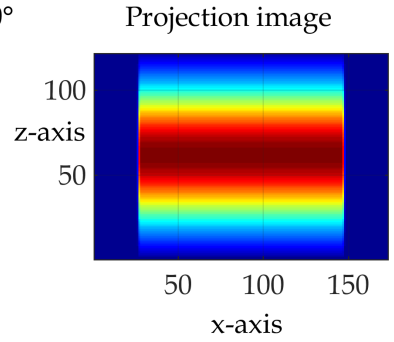

Projection image c)

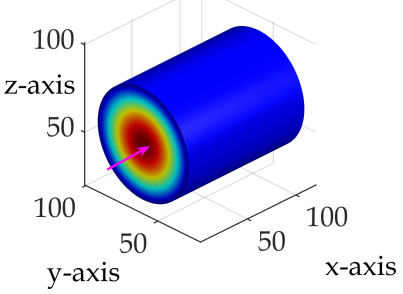

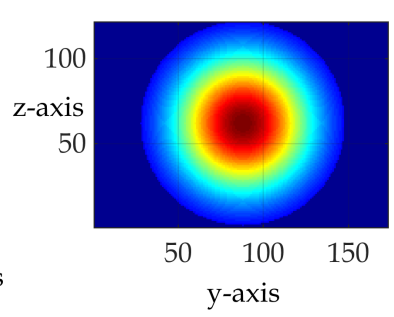

b)

d)

Figure 2. Examples of a 3D image of a squeezed trapped field in a cylindrical sample $(\mathbf{a}, \mathbf{c})$ and 2D projection images $(\mathbf{b}, \mathbf{d})$, orientation with respect to neutron flight direction (red arrow in $(\mathbf{a}, \mathbf{c})$ ) and projected intensities due to neutron spin depolarization $(\mathbf{b}, \mathbf{d})$. Red color $=4.6 \mathrm{mT}$, blue color $=0 \mathrm{mT}$.

With a so-called spin analyzer, the change of a neutron spin can be determined as a multiple of $\pi$. In the case of a trapped B field, $\mathrm{B}=\mathrm{B}_{\text {trap }}$ can be assumed to be given by the geometry of the sample. Its stray field $B_{\text {stray }}$ surrounding a (cylindrical) sample is, therefore, similar to that of a current carrying coil or magnetized rod. This stray field and the one of the spin analyzer also causes neutron spin to Larmor precessions $\left(\omega_{L}\right)$, which can be taken into account as follows: The stray field due to the spin analyzer was measured as a function of distance to sample position, and the calculated line integral of the stray field along this path (without a sample) yields a constant number of 37 (exactly 36.887) $2 \pi$ spin rotations which can be used as an instrumental offset. The stray field at the sample position at a distance of $18 \mathrm{~cm}$ is with $0.19 \mathrm{mT}$ more than an order of magnitude less than the trapped field in the sample [22]. Under these conditions, the total angle of Larmor precessions can be ascribed to the trapped field in the sample, only. The spin analyzer is transparent for spin parallel and opaque for spin anti-parallel orientation, so the final spin orientation with respect to the magnetization of the spin analyzer determines the contrast in the image. A 3D model of the trapped field can be found with $\mathrm{B}=\mathrm{B}(\mathrm{x}, \mathrm{y}, \mathrm{z})$, continuous and bounded by the sample, so the number of Larmor precession $\phi$ depends on the size of $\mathrm{B}$, only.

\subsection{Experimental Results}

More than 100 polarized neutron images have been recorded and evaluated. As mentioned above, one series was related to the un-treated $\mathrm{Nb}$ (" $\mathrm{Nb}_{\text {oxid }}$ ") sample, the other series when the same sample was further "treated" (" $\mathrm{Nb}_{b a k}$ ").

Figure $3\left(\mathrm{Nb}_{\text {oxid }}\right)$ and Figure $4\left(\mathrm{Nb}_{\text {bak }}\right)$ show two series of each 18 radiograms displaying the two-dimensional neutron spin depolarization images as functions of increasing AC offset field $\mathrm{B}_{\text {offset }}=1 \mathrm{mT} \ldots 5 \mathrm{mT}$ (horizontal images) and increasing AC-frequency (vertical images), in sample orientation $0^{\circ}$ (for orientation compare Figure 2). The change of color from red to blue in the images corresponds to a spin flip spin up (red color) $\rightarrow$ spin down (blue color), corresponding to red $\sim 0.9$ normalized intensity and blue $\sim 0.5$, and a trapped magnetic field $B=2.1 \mathrm{mT}$. The results are consistent with other flux trapping experiments with $\mathrm{Pb}$ [22-24]. In Figure 3, $\left(\mathrm{Nb}_{\text {oxid }}\right)$ the trapped field increases simply with increasing $B_{\text {offset }}$. If one use Equation (2), and $s=10 \mathrm{~mm}(\mathrm{~s}=$ diameter of the sample) a spin flip for a neutron wavelength $\lambda=0.32 \mathrm{~nm}$ occurs for $\mathrm{B}=2.1 \mathrm{mT}$ which indeed was observed for the center of the sample. In all rows in Figure $3\left(\mathrm{Nb}_{\text {oxid }}\right)$ and 
Figure $4\left(\mathrm{Nb}_{\text {bak }}\right)$, AC magnetic fields oscillate between $\pm 5 \mathrm{mT}$ with different frequencies $(0.1 \mathrm{~Hz}, 0.25 \mathrm{~Hz}$ and $0.5 \mathrm{~Hz})$ and increasing offset of $1 \mathrm{mT} \ldots 5 \mathrm{mT}$. Comparing images of a given offset field B in Figure 3, one observes for offset fields $4 \mathrm{mT}$ and $5 \mathrm{mT}$ an increase in field trap with increasing AC field; however, for offset fields $1 \mathrm{mT}-3 \mathrm{mT}$, non-dependency could be measured. A possible frequency dependency of trapped flux, $\mathrm{B}_{\text {trap }}=\mathrm{B}_{\text {trap }}(f)$ seemed to occur for offset fields larger than $3 \mathrm{mT}$; however, in contrast to expectations, the trapped field became larger instead of smaller.

$\mathrm{Nb}_{\text {oxid }}$, sample orientation $0^{\circ}$
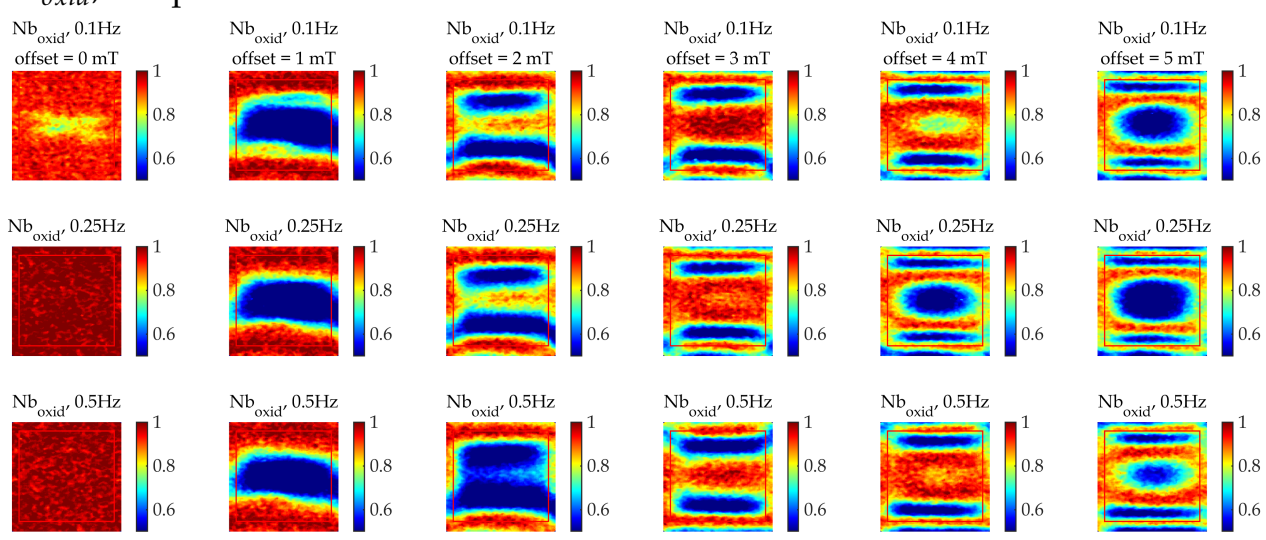

Figure 3. $\mathrm{Nb}_{\text {oxid }}$ sample: orientation $0^{\circ}, \mathrm{B}_{\text {offset }}=0 \mathrm{mT} \ldots 5 \mathrm{mT}$ (horizontal images) and increasing AC-frequency $\mathrm{f}=0.1 \mathrm{~Hz}, 0.25 \mathrm{~Hz}$ and $0.5 \mathrm{~Hz}$ (vertical images), blue $\sim 0.5$ and red $\sim 0.9$ normalized intensity, see text.

After the sample was annealed at $1400{ }^{\circ} \mathrm{C}$ and buffered chemically polished (BCP) $\left(\mathrm{Nb}_{\text {oxid }} \rightarrow \mathrm{Nb}_{\text {bak }}\right)$, a different behavior of the trapped fields was observed (see Figure 4 ). Here, one cannot observe a possible frequency dependence for a constant offset field of $4 \mathrm{mT}$ and/or $5 \mathrm{mT}$. The change (increase) of $\mathrm{B}_{\text {trap }}$ (color change) is rather observed for different offset fields. The increase of $B_{\text {trap }}$ is comprehensible if one keeps in mind that a spin rotation, from, e.g., spin up to spin down due to larger $B_{\text {trap, }}$, changes the image color (second and third row in Figure 4), or vice versa in the first row of Figure 4.

In the sample orientation of $90^{\circ}$ yields detailed views of the homogeneity of the trapped fields (see Figures 5 and 6), because once trapped, it rotates with the sample. In the sample orientation of $90^{\circ}$, all neutrons have the same path length in the sample (and in the trapped field, compare Figure 2), so different colors in an image indicate different spin depolarization due to different strengths of magnetic fields and thus different path integrals of B.ds. Note that colors in Figure 3 do not correlate to colors in Figure 5 and colors in Figure 4 not correlate to colors in Figure 6. In the $90^{\circ}$ sample orientation, $B_{\text {trap }}$ and its stray field are (nearly) parallel to the neutron flight direction. Its strength increases and decreases slowly for a spin polarized neutron along its path and favor adiabatic change of the spin direction which is much more likely than a spin rotation around $\vec{B}$. In order to quantify neutron spin interaction of the trapped field, it was calculated for each $B_{\text {trap }}=B_{\text {trap }}(f$, offset $)$ in $0^{\circ}$ sample orientation, which provided clearer information than the $90^{\circ}$ sample orientation (not shown here). 
$\mathrm{Nb}_{\text {bak }}$, sample orientation $0^{\circ}$
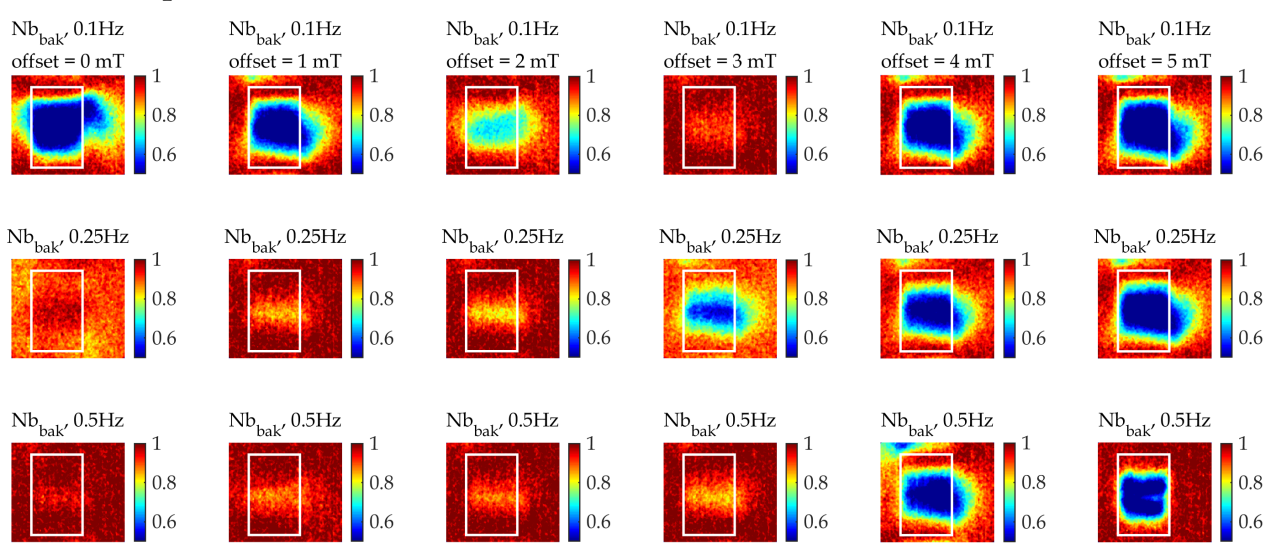

Figure 4. $\mathrm{Nb}_{b a k}$ sample orientation $0^{\circ}, \mathrm{B}_{\text {offset }}=0 \mathrm{mT} \ldots 5 \mathrm{mT}$ (horizontal images) and increasing AC-frequency $\mathrm{f}=0.1 \mathrm{~Hz}, 0.25 \mathrm{~Hz}$ and $0.5 \mathrm{~Hz}$ (vertical images), yellow boxes show the projected volumes of yellow circles in Figure 6, blue $\sim 0.5$ and red $\sim 0.9$ normalized intensity, see text.

$\mathrm{Nb}_{\text {oxid }}$, sample orientation $90^{\circ}$

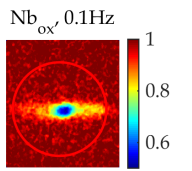

$\mathrm{Nb}{ }_{0 x^{\prime}} 0.25 \mathrm{~Hz}$

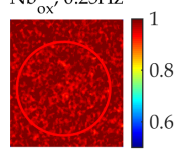

$\mathrm{Nb}{ }_{0 x^{\prime}} 0.5 \mathrm{~Hz}$

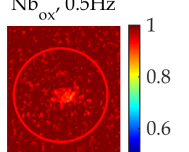

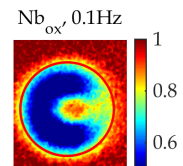

$\mathrm{Nb} \mathrm{ox}^{\prime}, 0.25 \mathrm{~Hz}$

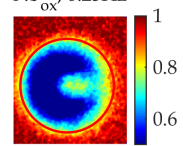

$\mathrm{Nb}{ }_{0 x^{\prime}} 0.5 \mathrm{~Hz}$

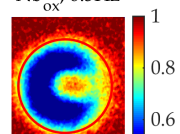

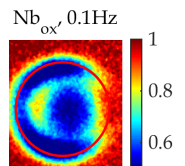

$\mathrm{Nb}_{\mathrm{ox}^{\prime}} 0.25 \mathrm{~Hz}$

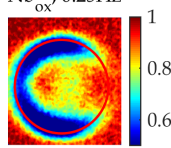

$\mathrm{Nb}_{\mathrm{ox}^{\prime}} 0.5 \mathrm{~Hz}$

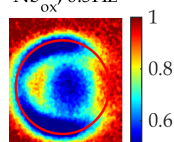

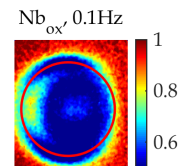

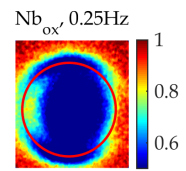

$\mathrm{Nb}, 0.5 \mathrm{~Hz}$

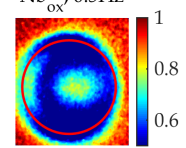

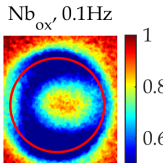

$\mathrm{Nb}_{\mathrm{ox}^{\prime}} 0.25 \mathrm{~Hz}$

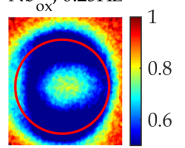

$\mathrm{Nb}{ }^{\prime} 0.5 \mathrm{~Hz}$

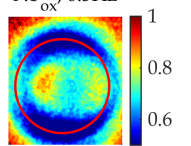

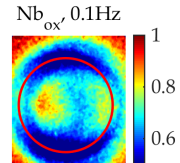

$\mathrm{Nb}, 0.25 \mathrm{~Hz}$

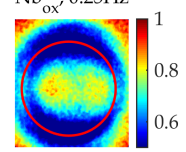

$\mathrm{Nb}, 0.5 \mathrm{~Hz}$

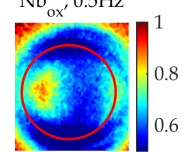

Figure 5. $\mathrm{Nb}_{\text {oxid }}$ sample orientation $90^{\circ}, \mathrm{B}_{\text {offset }}=0 \mathrm{mT} \ldots 5 \mathrm{mT}$ (horizontal images) and increasing AC-frequency $\mathrm{f}=0.1 \mathrm{~Hz}, 0.25 \mathrm{~Hz}$ and $0.5 \mathrm{~Hz}$ (vertical images), blue $\sim 0.5$ and red $\sim 0.9$ normalized intensity, see text.

$\mathrm{Nb}_{\text {bak }}$, sample orientation $90^{\circ}$
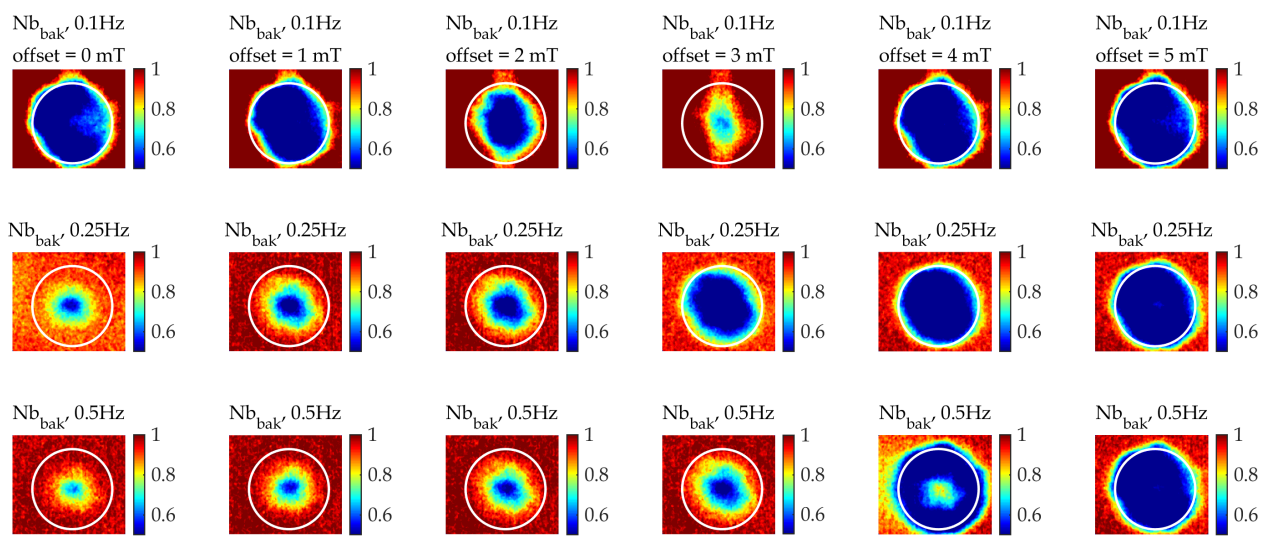

Figure 6. $\mathrm{Nb}_{b a k}$ sample orientation $=90^{\circ}, \mathrm{B}_{\text {offset }}=0 \mathrm{mT} \ldots 5 \mathrm{mT}$ (horizontal images) and increasing AC-frequency $\mathrm{f}=0.1 \mathrm{~Hz}$, and $0.25 \mathrm{~Hz}$ and $0.5 \mathrm{~Hz}$ (vertical images), blue $\sim 0.5$ and red $\sim 0.9$ normalized intensity, see text. 
The best agreement with the experimental data was found under the assumption that the trapped field behaves similar to that of a magnetized coil. The 2D projection images of the 3D trapped fields (see Figure 2) were calculated and compared with the experimental ones, using line plots, as is shown in Figures 7 and 8. As can be seen, the trapped field is always larger than the nominal size of the external field, due to the AC contribution. This behavior suggests the assumptions of an homogeneously trapped field and of a possible frequency dependency of field trap, which is best seen for the offset = $4 \mathrm{mT}$ and $5 \mathrm{mT}$; however, a general frequency dependence can not yet proven.

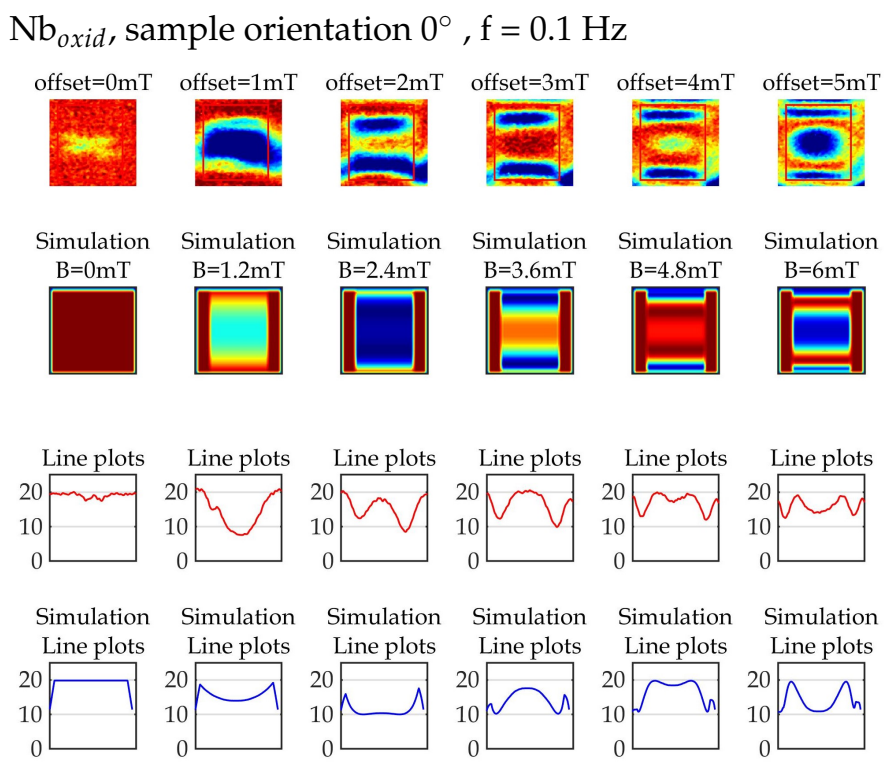

Figure 7. $\mathrm{AC} f=0.1 \mathrm{~Hz}$ : trapped field experiments and calculations, sample $=\mathrm{Nb}_{\text {oxid }}$, trapped field $\mathrm{B}=\mathrm{B}_{\text {trap }}$ as function of offset field $\mathrm{B}=0 \mathrm{mT} \ldots 5 \mathrm{mT}$ (compare first row in Figure 3), blue $\sim 0.5$ and red $\sim 0.9$ normalized intensity, line plots correspond to projections onto the vertical axis of the $2 \mathrm{D}$ plots within the central areas.

$\mathrm{Nb}_{\text {oxid }}$, sample orientation $0^{\circ}, \mathrm{f}=0.5 \mathrm{~Hz}$

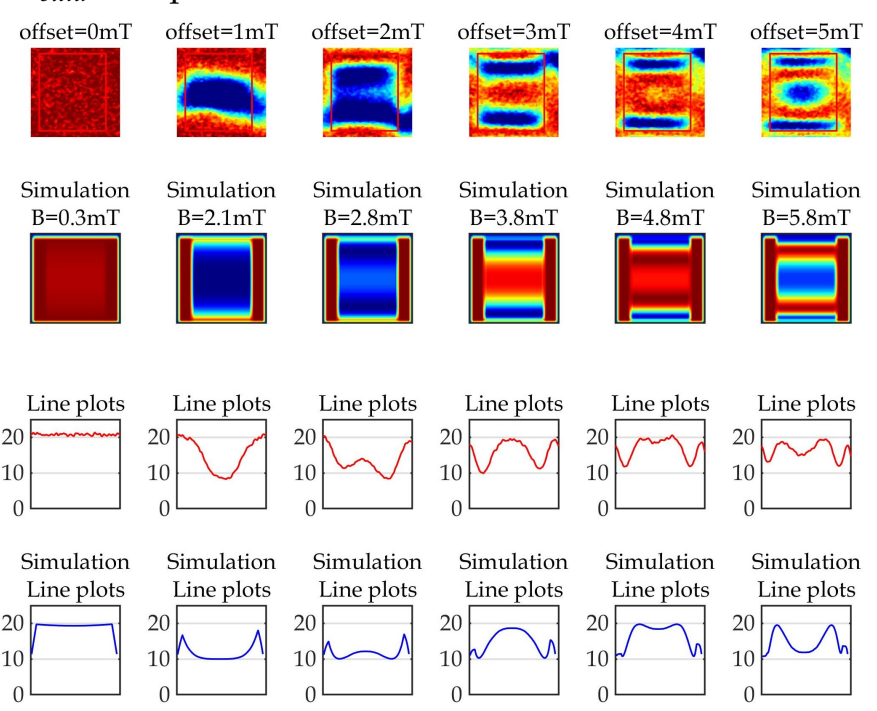

Figure 8. $\mathrm{AC} \mathrm{f}=0.5 \mathrm{~Hz}, \mathrm{Nb}_{\text {oxid }}$, trapped field experiments and calculations, trapped field as function of offset field $\mathrm{B}=0 \mathrm{mT} \ldots 5 \mathrm{mT}$ (compare third row in Figure 3), blue $\sim 0.5$ and red $\sim 0.9$ normalized intensity, line plots correspond to projections onto the vertical axis of the 2D plots within the central areas. 
It was necessary to prove whether a frequency dependence of magnetic flux trapping another calculation was applied to the data. If flux trapping depends on applied AC fields, then the size of trap in a given sample volume is supposed to change with frequency. For this purpose, the central area (pixel field) of all images were selected and the size of the trapped flux was determined as a function of the AC field and frequency. If there is a frequency dependency, the magnitude of the trapped field in the sample must change with AC-frequency for a given offset field. For this reason, 32 pixel $\times 32$ pixel $=1024$ pixel $\left(1.37 \mathrm{~mm} \times 1.27 \mathrm{~mm}=1.89 \mathrm{~mm}^{2}\right)$ of each image in Figure $3\left(\right.$ sample orientation $\left.0^{\circ}\right)$ and in Figure 5 (sample orientation $90^{\circ}$ ) were selected from the central area and the intensities were summed up (integrated). If one plots integrated intensity of the trapped field of the central area (1024 pixel) of the images in Figure $3\left(\mathrm{Nb}_{\text {oxid }}\right.$, sample orientation $\left.=0^{\circ}\right)$ and Figure $5\left(\mathrm{Nb}_{\text {oxid }}\right.$, sample orientation $\left.=90^{\circ}\right)$ vs. the offset field for $\mathrm{f}=0.1 \mathrm{~Hz}, 0.25 \mathrm{~Hz}$ and $0.5 \mathrm{~Hz}$, corresponding graphs must remarkably differ from each other. Figures 9 and 10 show the results, i.e., no f-dependency could be found, even if the central selected area of 1024 pixel was reduced to 4 pixel (not shown here).

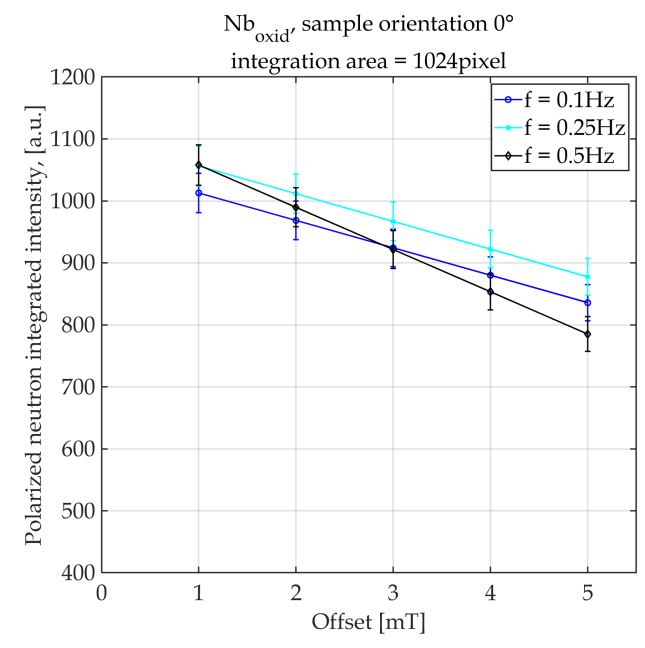

Figure 9. Integrated intensity vs. offset field, sample orientation $=0^{\circ}$. In the case of a frequency dependence, graphs must show for all offset fields remarkable differences of integrated intensities, i.e., linear fit lines should appear parallel and must differ in height from each other (see text).

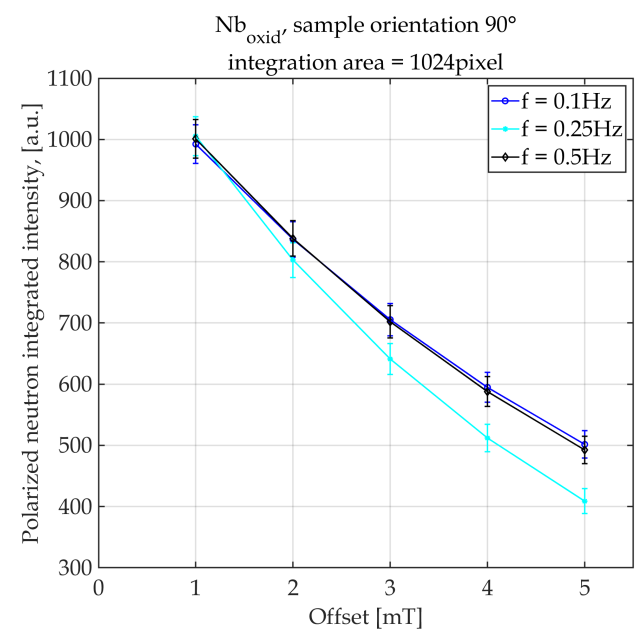

Figure 10. Integrated trapped field vs. offset field, sample orientation $=90^{\circ}$; the graphs must be clearly distinguishable from each other for all offset fields, see text.

The integrated intensities decrease due to an increase in flux trapping in the sample (increasing offset field), occurring for all applied AC fields, which causes the neutron spin 
to rotate from parallel (spin-up) to antiparallel (spin-down). Spin-down neutrons cannot pass through the spin analyzer and are not counted, since the spin analyzer is only transparent for spin-up neutrons. This behavior was calculated for both sample orientations.

In the case of $\mathrm{Nb}_{b a k}$, all images show a symmetrical flux trapping in both orientations, filling the sample with increasing frequencies, and furthermore, a much lower frequency and offset field dependence is observed. AC fields with increasing offset fields can produce trapped fields, but different to the $\mathrm{Nb}_{\text {oxid }}$ this effect is much weaker than in the case when the $\mathrm{Nb}$ sample was 'treated' $\left(\mathrm{Nb}_{b a k}\right.$, Figures 4 and 6$)$. There is no frequency dependence for offset fields of $4 \mathrm{mT}$ and $5 \mathrm{mT}$.

In the $90^{\circ}$ position, all paths through the sample are of equal path length. One observes a certain symmetric trapped flux centered around the rod axis filling the sample with increasing offset field (second and third row in Figure 6). There appears a weak (squeezed) trapped field seen in the $0^{\circ}$ sample orientation (Figure 4) and of course, also and better seen in the $90^{\circ}$ sample orientation (Figure 6). The trapped flux seems to dissipate in the sample with increasing offset field, i.e., it is first first squeezed and then homogeneously distributed.

In both sample orientations of $\mathrm{Nb}_{b a k}\left(0^{\circ}\right.$ and $\left.90^{\circ}\right)$ a trapped field of the order of $2 \mathrm{mT}$ appears for $\mathrm{f}=0.1 \mathrm{~Hz}$, (blue color indicates a $180^{\circ}$ spin flip), which vanishes for $\mathrm{f}=0.25 \mathrm{~Hz}$ and $\mathrm{f}=0.5 \mathrm{~Hz}$. This effect cannot stem from unknown external stray fields causing a neutron spin rotation from up to down, because this spin flip is observed in both sample orientation, after FC, and when the external field is switched off. A rotation of the sample also rotates the trapped field including its corresponding stray field. Therefore, this effect suggests a small field trap caused by the AC field, only.

Checking a possible frequency dependency of the trapped flux, the integrated intensities of the centers of the images in Figures 3 and $5\left(\mathrm{Nb}_{\text {oxid }}\right)$ and in Figures 4 and 6 , $\left(\mathrm{Nb}_{b a k}\right)$ were plotted as a functions of offset fields for frequencies $\mathrm{f}=0.1 \mathrm{~Hz}, 0.25 \mathrm{~Hz}$ and $0.5 \mathrm{~Hz}$. The graphs Figures 9 and $10\left(\mathrm{Nb}_{\text {oxid }}\right)$ and Figures 11 and $12\left(\mathrm{Nb}_{\text {bak }}\right)$ show the results. One hardly can deduce a real frequency dependence in either figure. In the case of $\mathrm{Nb}_{b a k}$, there seems to be some distinctiveness of the line graphs, because error bars are sometimes disjunctive, in both sample orientations, but the small trapped fields here differ by less than $2 \mathrm{mT}$ which cause spin rotation from up to down or vice versa. This explains the "big" difference in the integrated number of polarized neutrons for the offset fields $1 \mathrm{mT}$ and $2 \mathrm{mT}$.

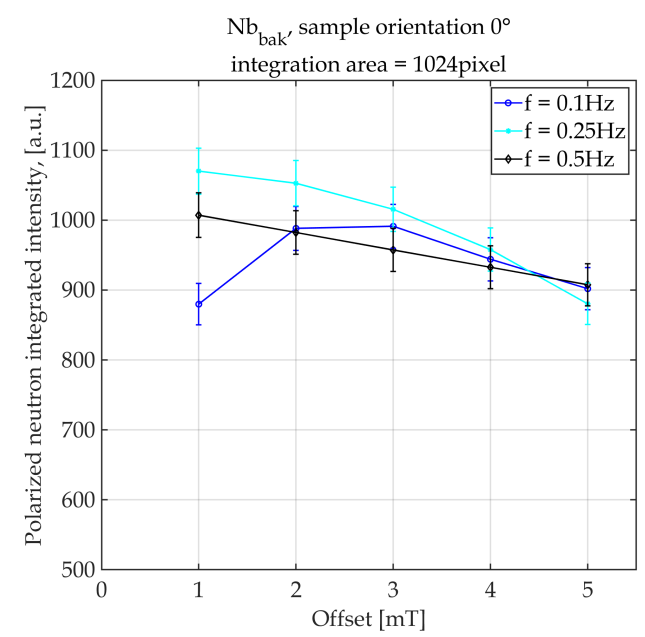

Figure 11. $\mathrm{Nb}_{b a k}(\mathrm{Nb}$ sample "treated"): the integrated intensity of central part of each image of Figure 4 (1024 pixel) was plotted as a function of offset field for frequencies $\mathrm{f}=0.1 \mathrm{~Hz}, 0.25 \mathrm{~Hz}$ and $0.5 \mathrm{~Hz}$ (see text). 


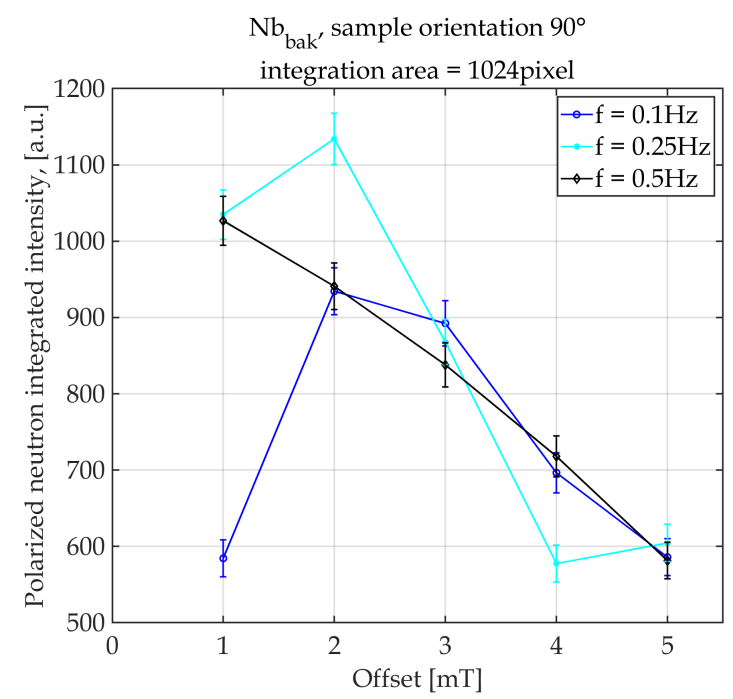

Figure 12. $\mathrm{Nb}_{b a k}(\mathrm{Nb}$ sample "treated"): the integrated intensity of central part of each image of Figure 6 (1024 pixel) was plotted as a function of offset field for frequencies $\mathrm{f}=0.1 \mathrm{~Hz}, 0.25 \mathrm{~Hz}$ and $0.5 \mathrm{~Hz}$ (see text).

As mentioned above, line graphs belonging to different frequencies must differ with respect to their integrated intensities for a given offset field. A possible frequency dependency of trapped flux would change the size of $\mathrm{B}_{\text {trap }}$ in this volume and thus the neutron depolarization of the incident polarized neutron beam. In all figures, Figures 9-12, no correlation or dependency can be found.

Another observation should be mentioned. In the $\mathrm{Nb}_{b a k}$, the AC field during FC created around the rod axis a cylindrical trapped flux which increases with the offset field, finally filling the sample homogeneously, seen in Figure 4, and better seen in Figure 6. However, the size of the increase seems to be independent of the frequency of the AC field.

\section{Conclusions}

Within an extended series of measurements, the influence of external magnetic AC fields with different offset fields on magnetic flux trapping during field cooling (FC) was investigated for a polycrystalline, heat-treated $\mathrm{Nb}$ sample of cylindrical shape (radius $=5 \mathrm{~mm}$, length $=10 \mathrm{~mm}$ ). Close to the transition temperature $\mathrm{T} \longrightarrow \mathrm{Tc}$ the mobility of flux lines should be enhanced by AC fields with different frequencies and offset amplitudes. The cooling time was chosen $1 \mathrm{~K} / \mathrm{min}$, which should be long enough around $\mathrm{T}_{\mathcal{C}}$ to influence the mobility of flux lines and thus flux trapping. Further cooling fixed this state in order to determine the trapped flux with polarized neutron imaging.

AC fields with different frequencies and amplitudes $\pm 5 \mathrm{mT}$ have been applied to the $\mathrm{Nb}$ sample during cooling from $\mathrm{T}=15 \mathrm{~K}$ down to $\mathrm{T}=5 \mathrm{~K}\left(\mathrm{~T}_{\mathcal{c}}=9.2 \mathrm{~K}\right)$ and at $\mathrm{T}=5 \mathrm{~K}$ and after switching off the external magnetic field, the size of flux trapping was measured with polarized neutron imaging.

When the $\mathrm{Nb}$ sample is left 'untreated' $\left(\mathrm{Nb}_{\text {oxid }}\right)$, different offset AC fields of $1 \mathrm{mT}$ ...5 $\mathrm{mT}$ increases flux trapping and, therefore, amplifies this unwanted impact instead of reducing it; this happens much less when the $\mathrm{Nb}$ sample is 'treated' $\left(\mathrm{Nb}_{b a k}\right)$. Here, one observes a small flux trapping symmetrically and centered around the rod axis. Due to increasing AC fields, the trapped flux appears more and more homogeneously distributed in the sample, especially in the case of $\mathrm{Nb}_{b a k}$ where, starting from the rod axis, the trapped flux dissipates to the whole volume. This remarkable behavior could be well observed with polarized neutrons; no other technique yields such position resolved (real) information about magnetic flux trapping in the bulk sample in the superconducting state, and these observations underline again the commonly known importance of a careful surface treatment in order to suppress magnetic field trapping during field cooling. The 
calculation of images of trapped flux under different conditions and comparison of these experimental ones yields good agreement for nearly all images.

As far as measurement technology (neutron imaging) is concerned, the result of the spin detection by the spin analyzer in this experiment is the same for both spin rotations, thus in this experiment one could not distinguish whether the trapped field showed in $+y$ or -y direction, one only could determine a certain increase or decrease in flux trapping. For our measurement, it was not important to know which orientation the trapped field had rather than to measure its size in the sample.

A comparison with results of publications on magnetic fields in $\mathrm{Nb}$, using neutron diffraction and neutron small angle scattering [9,10,36-38], shows the different approaches to obtain information about the bulk magnetism in superconductors. From the flux line densities, absolute values of the form factors were obtained, which allows us to determine local magnetic field distribution. The results of diffraction and small angle scattering are usually in the nm size range, about three orders of magnitude smaller than the spatial resolution of a current neutron image, and one obtains detailed (nm-large) local information, about the flux line density. In the measurements presented in this paper trapped magnetic fields are determined directly, i.e., visualized and quantified as functions of AC fields, applied during field cooling. A spatial resolution of $\mathrm{nm}$ is not useful at this point, since one is interested in a significant reduction of the flux lines and thus the trapped magnetic fields for the entire sample.

Therefore, the measurements and results obtained here are useful to test the flux line mobility under the influence of small AC fields during cooling from $\mathrm{T}>\mathrm{Tc}$ to $\mathrm{T}<\mathrm{Tc}$. Conservatively, one could say that the AC field has some function in homogenizing the trapped flux, which could indicate some frequency influence. This could be shown when the sample was 'treated'.

In summary, it can be stated that no evidence was found that magnetic flux trapping can be suppressed with small alternating magnetic fields during the cooling phase, especially when $T \sim T_{c}$, which shall drive out flux lines of the sample. For the values of cool-down speed and AC frequency tested here it seems that it only forces an homogeneous field distribution, which was best observed when the $\mathrm{Nb}$ sample was annealed and buffered chemically polished.

The parameter space explored in this initial study was somewhat limited and further experiments are required to finally assess if superimposing an AC field during transition can enhance the performance of SRF cavities. A potential experiment could involve keeping a sample below but close to its transition temperature for an extended time while applying an AC field. Furthermore, one could investigate so-called temperature cycles as were performed with $\mathrm{Pb}$ (without AC fields) to reduce the trapped flux [26].

In order to get deeper information about the time scale of arising flux trapping, polarized neutrons imaging can be a great help. The short transit time of a neutron and thus the interaction time with arising trapped magnetic field is less than $10 \mu \mathrm{s}$ for a path length of $10 \mathrm{~mm}$, so it could be used to probe time dependent flux trapping in thinner samples. For superconductivity, position-resolved information about processes in the bulk of samples, being in the Meissner state or Shubnikov state, with and without flux lines, can probably be best achieved with polarized neutron imaging.

Author Contributions: Conceptualization: W.T., O.K., T.J. Methodology: W.T., O.K., T.J. Software: W.T. Validation: W.T., O.K., T.J. Writing—original draft preparation: W.T., O.K., T.J. Funding acquisition: W.T. All authors have read and agreed to the published version of the manuscript.

Funding: This research was funded by the Federal Ministry of Education and Research-BMBF, project 05 K10FK1.

Institutional Review Board Statement: Not applicable.

Informed Consent Statement: Not applicable. 
Acknowledgments: We are very grateful to Johannes Nicol, Luisa Riik, Ralf Ziesche and Henning Höppner for their help with the measurements at the Berlin research reactor BER II, to the HelmholtzZentrum Berlin Wannsee for the support with its infrastructure and of course to the BMBF (Federal Ministry of Education and Research) Germany. This work was part of the BMBF project 05 K10FK1.

Conflicts of Interest: The authors declare no conflict of interest.

Remark: The PONTO II instrument operated by the UAS Berlin had to be finally dismantled due to the shutdown of the BER II (Berlin Experimental Reactor II) in 2019.

\section{References and Note}

1. Ciovati, G.; Gurevich, A. Evidence of high-field radio-frequency hot spots due to trapped vortices in niobium cavities. Phys. Rev. ST Accel. Beams 2008, 11, 122001. [CrossRef]

2. Aull, S.; Kugeler, O.; Knobloch, J. Trapped magnetic flux in superconducting niobium samples. Phys. Rev. ST Accel. Beams 2012, 15, 062001. [CrossRef]

3. Vogt, J.-M.; Kugeler, O.; Knobloch, J. Impact of cool-down conditions at T c on the superconducting rf cavity quality factor. Phys. Rev. ST Accel. Beams 2013, 16, 102002. [CrossRef]

4. Romanenko, A.; Grassellino, A.; Crawford, A.; Sergatskov, D.; Melnychuk, O. Ultra-high quality factors in superconducting niobium cavities in ambient magnetic fields up to $190 \mathrm{mG}$. Appl. Phys. Lett. 2014, 105, 234103. [CrossRef]

5. Vogt, J.-M.; Kugeler, O.; Knobloch, J. High-Q operation of superconducting rf cavities: Potential impact of thermocurrents on the rf surface resistance. Phys. Rev. ST Accel. Beams 2015, 18, 042001. [CrossRef]

6. Eichhorn, R.; Daly, C.; Furuta, F.; Ganshyn, A.; Ge, M.; Gonnella, D.; Hall, D.; Ho, V.; Hofstaetter, G.H.; Liepe, M.; et al. Cornell's Main Linac Cryomodule for the Energy Recovery Linac Project. Phys. Rev. Accel. Beams 2016, 19, 012001. [CrossRef]

7. Posen, S.; Checchin, M.; Crawford, A.C.; Grassellino, A.; Martinello, M.; Melnychuk, O.S.; Romanenko, A.; Sergatskov, D.A.; Trenikhina, Y. Efficient expulsion of magnetic flux in superconducting radiofrequency cavities for high Q 0 applications. J. Appl. Phys. 2016, 119, 213903. [CrossRef]

8. Koszegi, J.; Kugeler, O.; Abou-Ras, D.; Knobloch, J.; Schaefer, R. A magneto-optical study on magnetic flux expulsion and pinning in high-purity niobium. J. Appl. Phys. 2017, 122, 173901. [CrossRef]

9. Mühlbauer, S.; Pfleiderer, C.; Böni, P.; Laver, M.; Forgan, E.M.; Fort, D.; Keiderling, U.; Behr, G. Morphology of the superconducting vortex lattice in ultrapure niobium. Phys. Rev. Lett. 2009, 102, 136408. [CrossRef]

10. Reimann, T.; Schulz, M.; Mildner, D.F.R.; Bleuel, M.; Brulet, A.; Harti, R.P.; Benka, G.; Bauer, A.; Böni, P. Domain formation in the type-II/1 superconductor niobium: Interplay of pinning, geometry, and attractive vortex vortex interaction. Phys. Rev. B 2017, 96, 144506. [CrossRef]

11. Schmitz, B.; Koszegi, J.; Alomari, K.; Kugeler, O.; Knobloch, J. Magnetometric mapping of superconducting RF cavities. Rev. Sci. Instrum. 2018, 89, 054706. [CrossRef] [PubMed]

12. Knobloch, J. RF Superconductivity for Accelerators, Padamsee, H. In Proceedings of the 8th Workshop on RF Superconductivity, Padova, Italy, 6-10 October 1997; pp. 337-344.

13. Benvenuti, C.; Calatroni, S.; Campisi, I.; Darriulat, P.; Durand, C.; Peck, M.; Russo, R.; Valente, A.-M. Niobium sputter-coated copper resonators. In Proceedings of the 8th Workshop on RF Superconductivity, Padova, Italy, 6-10 October 1997; pp. 331-335.

14. Aull, S. Investigation of trapped magnetic flux in superconducting niobium samples. In Diplomarbeit; Humboldt-Universitaet Berlin: Berlin, Germany, 2011.

15. Ciovati, G.; Gurevich, A. Measurement of RF losses due to trapped flux in a large grain Niobium cavity. In Proceedings of the 13th Workshop on RF Superconductivity, Berlin, Germany, 14-19 October 2007; pp. 132-136

16. Kugeler, O.; Neumann, A.; Anders, W.; Knobloch, J. Adapting TESLA technology for future cw light sources using HoBiCaT. Rev. Sci. Instrum. 2010, 81, 074701. [CrossRef] [PubMed]

17. Aull, S.; Ebrahimi, O.; Karakas, N.; Knobloch, J.; Kugeler, O.; Treimer, W. Suppressed Meissner-effect in Niobium: Visualized with polarized neutron radiography. J. Phys. Conf. Ser. 2012, 340, 012001. [CrossRef]

18. Krzyzagorski, M. "Neutronenradiographien von Gefangenen Magnetfeldern in Polykristallinen Niobproben" (Neutron Radiographs of Trapped Magnetic Fields in Polycrystalline Niobium Samples). Bachelor's Thesis, University of Applied Sciences, Berlin, Germany, 2016.

19. Treimer, W.; Hilger, A.; Kardjilov, N.; Strobl, M. Review about old and new imaging signals for neutron computerized tomography. Nucl. Instrum. Methods Phys. Res. Sect. A Accel. Spectrometers Detect. Assoc. Equip. 2005, 542, 367-375. [CrossRef]

20. Kardjilov, N.; Manke, I.; Strobl, M.; Hilger, A.; Treimer, W.; Meissner, M.; Krist, T.; Banhart, J. Three-dimensional imaging of magnetic fields with polarized neutrons. Nat. Phys. 2008, 4, 399-403. [CrossRef]

21. Schulz, M.; Neubauer, A.; Masalovich, S.; Muehlbauer, M.; Calzada, E.; Schillinger, B.; Pfeiderer, C.; Böni, P. Towards a tomographic reconstruction of neutron depolarization data. J. Phys. Conf. Ser. 2010, 211, 012025. [CrossRef]

22. Treimer, W.; Ebrahimi, O.; Karakas, N.; Prozorov, R. Polarized neutron imaging and three-dimensional calculation of magnetic flux trapping in bulk of superconductors. Phys. Rev. B 2012, 85, 184522. [CrossRef]

23. Treimer, W.; Ebrahimi, O.; Karakas, N. Observation of partial Meissner effect and flux pinning in superconducting lead containing non-superconducting parts. Appl. Phys. Lett. 2012, 101, 162603-1-162603-4. [CrossRef] 
24. Treimer, W. Radiography and tomography with polarized neutrons. J. Magn. Magn. Mater. 2014, 350, 188-198. [CrossRef]

25. Valsecchi, J.; White, J.S.; Bartowiak, M.; Treimer, W.; Kim, Y.; Lee, A.W.; Gokhfeld, D.M.; Hari, R.P.; Morgano, M.; Strobl, M.; et al. Visualization of compensating currents in type-II/1 superconductor via high field cooling. Appl. Phys. Lett. 2020, 116, 192602. [CrossRef]

26. Treimer, W. Handbook of Advanced Nondestructive Evaluation; Chapter 34 "Neutron Radiography and Tomography"; Springer International Publishing: Cham, Switzerland, 2019; pp. 1217-1299.

27. Treimer, W.; Ebrahimi, O.; Karakas, N. Imaging of quantum mechanical effects in superconductors by means of polarized neutron radiography. Phys. Procedia 2013, 43, 243-253. [CrossRef]

28. Mezei, F. Neutron spin echo: A new concept in polarized thermal neutron techniques. Z. Phys. Phys. A Hadron. Nucl. 1972, 255, 146-160. [CrossRef]

29. Badurek, G. NESY Winterschool 2007. Available online: http://planner2011.unileoben.ac.at/fileadmin/shares/planner2011/ docs/private/29-Badurek.pdf (accessed on 20 March 2021).

30. Schärpf, O. Available online: http:/ / host-82-135-31-182.customer.m-online.net/neutronpol.pdf (accessed on 20 March 2021).

31. NIST 'Neutron Scattering Length and Cross Sections'. Available online: https://physics.nist.gov/cuu/Constants/index.html (accessed on 20 March 2021)

32. NIST Center for Neutron Research. Available online: https://ncnr.nist.gov/resources/n-lengths/elements/fe.html (accessed on 20 March 2021).

33. Junginger, T.; Abidi, S.H.; Maffett, R.D.; Buck, T.; Dehn, M.H.; Gheidi, S.; Kiefl, R.; Kolb, P.; Storey, D.; Thoeng, E.; et al. Field of first magnetic flux entry and pinning strength of superconductors for $\mathrm{rf}$ application measured with muon spin rotation. Phys. Rev. Accel. Beams 2018, 21, 032002. [CrossRef]

34. Treimer, W.; Ebrahimi, O.; Karakas, N.; Seidl, S.O. PONTO-An instrument for imaging with polarized neutrons. Nucl. Instr. Methods Phys. Res. A 2011, 651, 53-56. [CrossRef]

35. W. R. Hendee and E. R. Ritenour, 'Medical Imaging Physics', chapter 18, p 456, Mosby Year Book, 3rd.edition (1992).

36. Schelten, J.; Ullmaier, H.; Lippmann, G. Local magnetic field distributions in superconducting niobium at $4.2 \mathrm{k}$ by neutron diffraction. Local magnetic field distributions in superconducting niobium at $4.2 \mathrm{~K}$ by neutron diffraction. Z. Phys. A Hadron. Nucl. 1972, 253, 219-231. [CrossRef]

37. Weber, H.W.; Schelten, J.; Lippmann, G. Microscopic Magnetic Field Distribution in Superconducting Niobium Single Crystals in the 'Dirty Limit'. Phys. Status Solidi 1973, 57, 515-522. [CrossRef]

38. Schelten, J.; Ullmaier, H.; Lippmann, G.; Schmatz, W. Low Temperature Physics-LT 13; Timmerhaus, K.D., O'Sullivan, W.J., Hammel, E.F., Eds.; Springer: Boston, MA, USA, 1974; pp. 54-63. 